\title{
Trifolitoxin Production in Rhizobium etli Strain CE3 Increases Competitiveness for Rhizosphere Colonization and Root Nodulation of Phaseolus vulgaris in Soil
}

\author{
Eduardo A. Robleto, Alexandra J Scupham, and Eric W. Triplett \\ Department of Agronomy, the Center for the Study of Nitrogen Fixation, and the Brock Institute for Envi- \\ ronmental Microbiology, University of Wisconsin-Madison, 1575 Linden Dr., Madison 53706 U.S.A. \\ Received 26 September 1996. Accepted 6 December 1996.
}

\begin{abstract}
The effect of trifolitoxin (TFX) production by Rhizobium etli on rhizosphere colonization and competition for nodulation in soil conditions was determined. TFX is a potent peptide antibiotic made by Rhizobium leguminosarum bv. trifolii T24 that inhibits many $\alpha$-proteobacteria (E. W. Triplett, B. T. Breil, and G. A. Splitter, Appl. Environ. Microbiol. 60:4163-4166, 1994). Seeds of Phaseolus vulgaris were inoculated with a TFX-sensitive reference strain and either of two isogenic strains that differ only in their ability to produce TFX. The pair of strains were inoculated at different ratios in sterile and nonsterile soil. The representation of the strains in the rhizosphere and nodules was determined at $96 \mathrm{~h}$ after inoculation and 3 weeks after planting, respectively. The TFX-producing strain was significantly more competitive for both phenotypes versus the TFX-sensitive strain, compared with the TFX-nonproducing strain versus the TFX-sensitive strain. These results show that nodule occupancy by inoculant strains, often displaced from the nodules by indigenous strains, can be increased by addition of the TFX production phenotype to $R$. etli in plants grown in either sterile or nonsterile soil. Also, this work shows the efficacy of the TFX system for the first time on a legume host with determinant nodules.
\end{abstract}

Additional keywords: legumes, peptide antibiotics.

A major limiting factor for the use of Rhizobium inoculant in legume production is the inability of inoculum strains to nodulate in the presence of indigenous rhizobia (for reviews see Dowling and Broughton 1986; Bottomley 1992; Triplett and Sadowsky 1992; Streeter 1994; Maier and Triplett 1996). This problem becomes of agronomic interest when the indigenous strains that prevent the inoculant strains from occupying the nodules fix nitrogen poorly or inefficiently. As a result, there is no yield response to inoculation.

Several strategies have been described to address the competitiveness problem (for review see Maier and Triplett 1996). In this laboratory, the ability of inoculant strains to produce a potent peptide antibiotic, trifolitoxin (TFX), has been de-

Corresponding author: Eric W. Triplett

E-mail: ewtriple@ facstaff.wisc.edu scribed as a possible means to prevent nodulation by TFXsensitive rhizobia (Triplett and Barta 1987; Triplett 1990). TFX is a ribosomally encoded, post-translationally modified, peptide antibiotic (Lethbridge 1989; Breil et al. 1993, 1996). TFX inhibits growth of all Rhizobium spp. tested to date as well as other species of the $\alpha$-proteobacteria (Triplett et al. 1994). TFX is produced by $R$. leguminosarum bv. trifolii strain T24, which induces ineffective nodules on clover roots (Schwinghamer and Belkengren 1968). Thus, T24 is not a useful inoculant strain to address the competition problem. Genes for TFX production and resistance have been transferred by conjugation to several species of $\alpha$-proteobacteria including all Rhizobium spp. tested (Triplett et al. 1994, 1989). To study the effects of TFX production without plasmid loss, Triplett (1990) constructed a strain that contained the TFX genes inserted in the chromosome of $R$. leguminasrum bv. trifolii-inoculated clover plants grown on Jensen's agar, and found that a TFX-producing strain occupied significantly more nodules than a near isogenic nonproducing strain when coinoculated with a TFX-sensitive strain.

Bosworth et al. (1993) showed differences in TFX production in sterile soils, with the highest production in a soil with high organic matter. TFX production was not observed in nonsterile soils where TFX was rapidly degraded. Whether TFX production increases the competitiveness of a strain in either sterile or nonsterile soil remained unresolved. In this paper, we show that addition of $t f x$ to $R$. etli increases its ability to compete for rhizosphere colonization and to compete for root nodulation in sterile and nonsterile soil. Several experiments have shown that $t f x$ can confer increased nodulation competitiveness in $R$. leguminosarum bv. trifolii, but all of these experiments were done with clover plants cultured in an artificial, sterile growth medium (Triplett and Barta 1987; Triplett 1988, 1990).

\section{RESULTS}

Competition of a TFX-producing strain in the rhizosphere in sterile soil.

The proportion of each of the strains in the rhizosphere $96 \mathrm{~h}$ after planting is depicted in two ways for plants inoculated with mixtures at different ratios of CE3(pFPMZ), the TFX- 
sensitive strain used as the reference strain for these experiments, and either a TFX-producing strain, CE3(pT2TFXK), or a TFX-nonproducing strain, CE3(pT2TX3K) (Figs. 1 and 2A). Figure 1 shows the number of CFU isolated from the rhizosphere of root systems from 4-day-old plants. The TFXsensitive strain colonized the bean roots at higher numbers than the TFX-nonproducing strain even when the TFXnonproducing strain was in much higher numbers in the inoculum (Fig. 1A). In contrast, the TFX-producing strain was present in larger numbers in the rhizosphere than the TFXsensitive strain when the two strains were inoculated at an approximately 1:1 ratio 4 days earlier (Fig. 1 b).

Figure 2A shows a simplified representation of the rhizosphere experiment data shown in Figure 1. The increased rhizosphere colonization conferred by the TFX production phenotype is presented in Figure 2A as the proportion of either $\mathrm{CE} 3(\mathrm{pT} 2 \mathrm{TFXK})$ or CE3(pT2TX3K) relative to the reference strain, CE3(pFPMZ). In comparable inoculum ratios, representation of the TFX-producing strain was significantly higher than the representation of the TFX-nonproducing strain except when their representations in the inoculum were near zero (Fig. 2A). To obtain a $50 \%$ representation of the TFX-

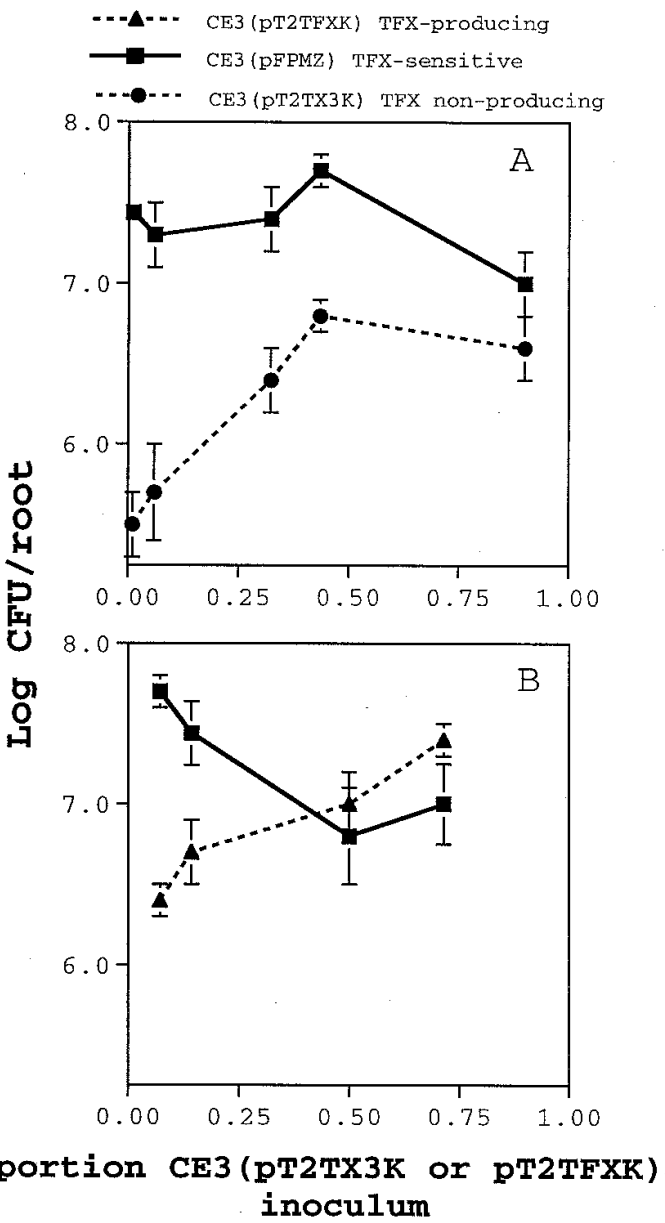

Fig. 1. Number of CFU isolated from the rhizospheres of 4-day-old root systems inoculated with the reference strain CE3(pFPMZ) (trifolitoxin [TFX]-sensitive) and either (A) TFX-nonproducing CE3(pT2TX3K) or (B) TFX-producing CE3(pT2TFXK). producing strain in the rhizosphere, the TFX-sensitive strain required a sevenfold excess in the inoculum. In contrast, a ninefold excess of the TFX-nonproducing strain in the inoculum was sufficient to reach $35 \%$ representation in the rhizosphere. The highest representation in the rhizosphere by the TFX-producing strain was $70 \%$, significantly different from the highest representation (35\%) in the rhizosphere reached by the TFX-nonproducing strain (Fig. 2A).
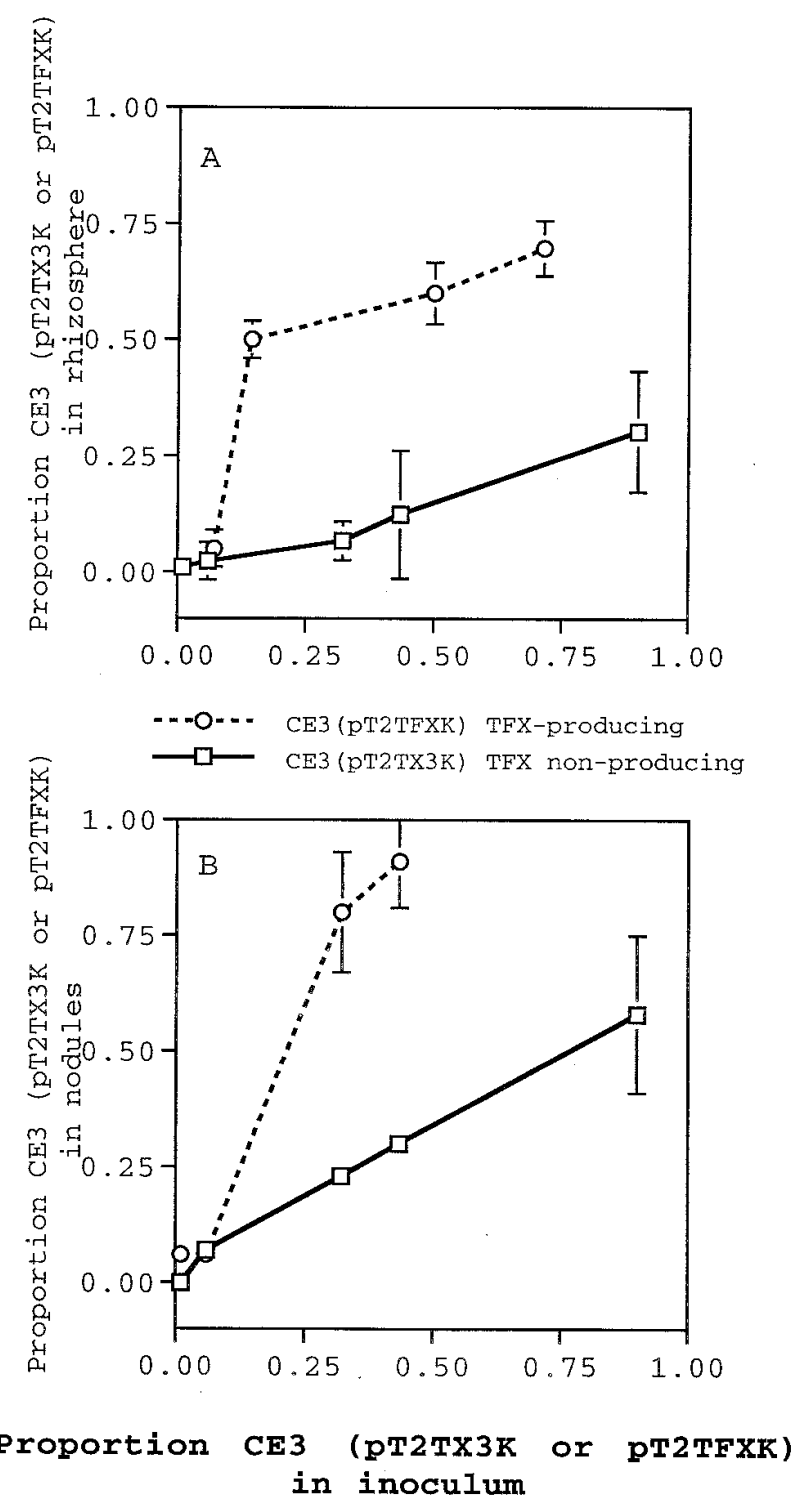

Fig. 2. Representation of trifolitoxin (TFX)-nonproducing (CE3 (pT2TX3K)) and TFX-producing (CE3(pT2TFXK)) strains inoculated at different ratios with the reference strain CE3(pFPMZ) (TFX-sensitive) in (A) the rhizosphere and (B) nodules in sterile soil. Proportion of the strain in the inoculum, rhizosphere, or nodules was estimated with the following formulas adapted from Amarger and Lobreau (1982): TFXnp / $($ TFXnp + TFXs); or TFXp / (TFXp + TFXs); where TFXnp $=$ TFXnonproducing, TFXs = TFX-sensitive, and TFXp = TFX-producing. Means of representation in rhizosphere and nodule occupancy based on five and 10 replications, respectively. Bars in graph indicate standard error values, error values are small where not seen. 


\section{Competition of a TFX-producing strain in root nodules in sterile soil.}

Presence of $t f x A B C D E F G$ improved competition for nodulation. A $25 \%$ representation of the TFX-producing strain in the inoculum (compared with that of the TFX-sensitive strain) was sufficient to reach $50 \%$ nodule occupancy. Furthermore, it took less than a $50 \%$ representation in the inoculum of the TFX-producing strain to reach $88 \%$ of nodule occupancy (Fig. 2B). In contrast, in comparable inoculation mixtures with TFX-sensitive and TFX-nonproducing strains, nodule occupancy of the TFX-nonproducing strain was significantly lower than that of its TFX-producing counterpart. A ninefold excess of the TFX-nonproducing strain was necessary to reach a $50 \%$ nodule occupancy. Inoculation of a mixture containing a 1:1 ratio of the TFX-nonproducing and reference strains resulted in $28 \%$ of nodules occupied by the TFX-nonproducing strain (Fig. 2B).

The representation in the rhizosphere and nodule occupancy of the strains have a similar response. These results suggest that production of TFX takes place early after inoculation and provides a competitive advantage in competition for nodulation.

\section{Competition of a TFX-producing strain in root nodules in nonsterile soil.}

Nodulation by indigenous rhizobia was negligible owing to the low population size of these bacteria in soil and the large number of rhizobia inoculated on the plants. The addition of tf $x A B C D E F G$ to $R$. etli strain CE3 increased its ability to compete for nodulation in nonsterile soil conditions. The TFX-producing strain reached significantly higher nodule occupancies than the TFX-nonproducing strain at comparable representations in the inoculum (Fig. 3). The TFX-producing strain required only a $15 \%$ representation in the inoculum to

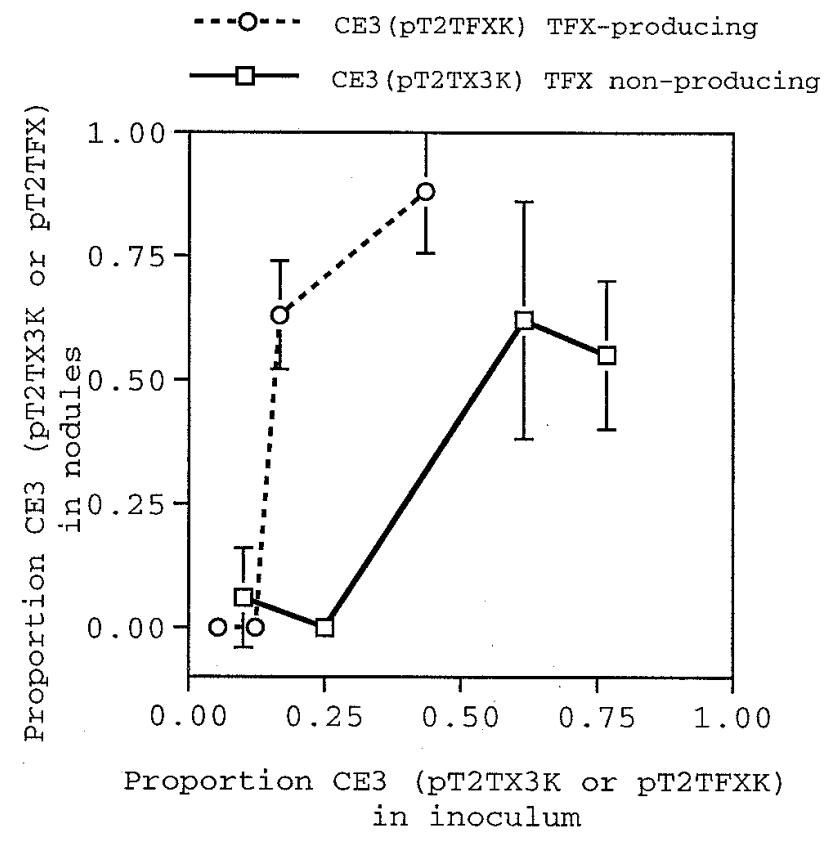

Fig. 3. Representation of trifolitoxin (TFX)-nonproducing (CE3 (pT2TX3K)) and TFX-producing (CE3(pT2TFXK)) strains inoculated at different ratios with the reference strain, TFX-sensitive CE3(pFPMZ) in nodules in nonsterile soil. Proportions were estimated as in Figure 2. reach $60 \%$ nodule occupancy, whereas the TFX-nonproducing strain required well over a $50 \%$ representation in the inoculum to occupy $50 \%$ of the nodules. The highest nodule occupancy by the TFX-producing strain was $80 \%$, which corresponded to the inoculation mixture with $45 \%$ TFX-producing strain and 55\% TFX-sensitive strain. In contrast, nodule occupancies higher than $50 \%$ by the TFX-nonproducing strain were only obtained with inoculation mixtures in which more than $50 \%$ of the inoculum was this strain (Fig. 3). These results suggest that TFX production by $R$. etli increased the ability to compete for nodulation in nonsterile soil conditions.

\section{Regression analysis.}

To determine numerically whether TFX production provides an advantage in competition for nodulation, competitiveness indices between the TFX-sensitive strain and either the TFX-nonproducing strain or TFX-producing strain were estimated as described by Beattie and Handelsman (1989b). With the means of the treatments from the competition for nodulation experiments we estimated competition indices between these strains (Table 1). The competitive indices for the TFX-sensitive strain in relation to the TFX-nonproducing strain are above zero for the sterile and nonsterile soils. This indicates that the TFX-nonproducing strain is less competitive than the TFX-sensitive strain. However, the negative values of the competitive indices for the TFX-sensitive strain in relation to the TFX-producing strain indicate that the presence of the TFX production phenotype conferred an increased ability to compete for nodulation (Table 1).

\section{DISCUSSION}

The effects of TFX production on the ability of $R$. etli strain CE3 to grow competitively in the rhizosphere and to compete for nodulation in sterile and nonsterile soil conditions were determined. These experiments are the first to illustrate an effect of $t f x$ on rhizosphere colonization (Fig. 2A). The significance of the efficacy of the TFX system in soil is important for the practical application of this technology to rhizobial inoculant strains used in agriculture, for our understanding of the role peptide antibiotics play in the soil environment, and for its support of the concept of a biased rhizosphere (Rossbach et al. 1994) to improve plant health and performance.

The presence of $t f x A B C D E F G$, rather than $t f x B^{\prime} C D E F G$, in $R$. etli enhanced the ability of the inoculum strain to colonize the rhizosphere, when compared with the reference strain in sterile soil (Fig. 2A). This work was done in a soil with high organic matter that contained $1.1 \times 10^{8}$ aerobic, culturable bacteria per $\mathrm{g}$ and $8.5 \times 10^{2}$ bean-nodulating rhizobia per $\mathrm{g}$.

Table 1. Competitiveness indices (CI) of a trifolitoxin (TFX)sensitive strain (CE3(pFPMZ)) in relation to TFX-nonproducing $(\mathrm{CE} 3(\mathrm{pT} 2 \mathrm{TX} 3 \mathrm{~K}))$ and TFX-producing (CE3(pT2TFXK)) strains in two soil conditions $\mathrm{a}^{\mathrm{a}}$

\begin{tabular}{lcc}
\hline & \multicolumn{2}{c}{ Competitiveness index } \\
\cline { 2 - 3 } Condition & CE3(pFPMZ): & CE3(pFPMZ): \\
CE3(pT2TX3K) & CE3(pT2TFXK) \\
\hline Sterile & +0.387 & -0.412 \\
Nonsterile & +0.493 & -1.002 \\
\hline
\end{tabular}

${ }^{a}$ CIs were calculated as described by Beattie and Handelsman (1989b). 
Previously, Bosworth et al. (1993) showed that this soil supports TFX production by $R$. leguminosarum bv. trifolii T24 after sterilization.

The evidence presented here shows that the addition of $t f x$ to Rhizobium can provide a competitive advantage in competition for nodulation in sterile and nonsterile soil. We showed that nodule occupancy of a TFX-producing strain was significantly higher than that of the TFX-sensitive reference strain when the strains were equally represented in the inoculum (Figs. 2B and 3). In contrast, competitiveness assays between the TFX-nonproducing strain and the TFX-sensitive strain showed that no competition advantage was gained by addition of a set of genes, tf $x B^{\prime} C D E F G$, that confers TFX resistance but not TFX production (Figs. $2 \mathrm{~B}$ and 3; Table 1). As we were unable to show TFX production in nonsterile soils previously (Bosworth et al. 1993), we expected that TFX would be degraded so rapidly in soil by proteases that any ecological roles of TFX production in soil would be difficult to demonstrate. However, the TFX production phenotype did enhance nodulation competitiveness in a nonsterile soil that contained a large, living bacterial population, so the observed rapid degradation of trifolitoxin does not prevent that antibiotic from exerting an important regulatory role in Rhizobium ecology. The TFX concentration around the TFX-producing cells and near the TFX-sensitive cells is apparently high enough to limit nodulation by the TFX-sensitive cell. However, the microbial population present in the nonsterile soil has some modulating effect on TFX, since the increase in nodulation competitiveness by the TFX-producing strain was more pronounced in the sterile soil than in the nonsterile soil.

We also found a correlation between the representations of the strains in the rhizosphere (Fig. 2). An increase in the representation of any of the strains tested in the rhizosphere coincided with an increase in nodule occupancy of the same strain. Marques-Pinto et al. (1974) developed a model to predict nodule occupancy based on the representation of the strains in the rhizosphere. Araujo et al. (1994) reported that a mutant reduced in competition for nodulation was also reduced in the extent of rhizosphere colonization, compared with the wildtype strain.

All strains used as inocula in this work contained recombinant plasmids for several reasons. First, we have found that stable integration of the $t f x$ region into most Rhizobium spp. is very difficult. Second, chromosomal insertion of $t f x$, when successful, provides much less TFX production than does plasmid-borne $t f x$, presumably because of multiple copies of the $t f x$ region when plasmid-borne. Third, plasmid-borne $t f x$ allowed us to easily construct near-isogenic strains, including a strain that lacked TFX production and another strain that was a useful reference strain. The most common problem associated with a plasmid-borne trait such as TFX production is that the plasmid is not stable during cell replication in the absence of selection pressure. This problem was circumvented here by the presence of the plasmid-partitioning locus from RK2 on each recombinant plasmid used in these experiments. Weinstein et al. (1992) showed that the presence of the partitioning locus conferred complete plasmid stability in Rhizobium both in culture and through nodulation. We confirmed the stability of pT2TFXK, pT2TX3K, and pFPMZ prior to conducting the experiments described here (data not shown).

The availability of $t f x$ on stable plasmids allows us to easily transfer the TFX production phenotype to any inoculum strain of Rhizobium or Sinorhizobium by conjugation. The level of TFX production conferred by pT2TFXK is high without any adverse affects on cell growth (data not shown). Although we have recently reported an important role in TFX production of another gene outside of the $t f x$ region in TFX production by $R$. leguminosarum bv. trifolii $\mathrm{T} 24$ (Breil et al. 1996), the addition of this gene, $t f u A$, to the $t f x$ cassette in pT2TFXK is unnecessary since all rhizobia exhibit high TFX production by addition of only $t f x A B C D E F G$. Either $t f u A$ is required for optimal TFX production only in T24 or tfuA is present in all rhizobia.

This work also shows the efficacy of the TFX system in a legume from the Phaseoleae tribe, Phaseolus vulgaris, that produces determinant root nodules. All of our previous competition experiments have been done with indeterminant nodule-forming species from the Trifolieae tribe, Trifolium repens or Medicago sativa. As predicted, the efficacy of the TFX system is independent of the host genotype provided that the microsymbiont infecting that host is TFX-sensitive. As TFX inhibits all Rhizobium or Sinorhizobium spp. tested to date (Triplett et al. 1994), the TFX production phenotype should improve the nodulation competitiveness of inoculants for all legumes infected by Rhizobium or Sinorhizobium.

\section{MATERIALS AND METHODS}

\section{Strains and plasmids.}

Strains and plasmids used in this study are shown in Table 2. Strains were kept in $15 \%$ glycerol at $-70^{\circ} \mathrm{C}$. Inoculum was grown from single colonies in yeast-mannitol broth (Beringer 1974) for $48 \mathrm{~h}$ at $28^{\circ} \mathrm{C}$ and $220 \mathrm{rpm}$ (Environ-Shaker, LabLine Instruments, Inc., Melrose Park, IL). Mixture inocula-

Table 2. Strains and plasmids used in this study

\begin{tabular}{lll}
\hline Strains/plasmids & Characteristics & Source or reference \\
\hline $\begin{array}{l}\text { Strains } \\
\text { Rhizobium etli CE3 }\end{array}$ & CFN42 derivative; Sm & Noel et al. 1986 \\
$\begin{array}{l}\text { Escherichia coli DH5 } \alpha \\
\text { Plasmids }\end{array}$ & recA; Nal & Bethesda Research Laboratories (Gaith ersburg, MD) \\
pBluescript & & \\
pFF1 & Cloning vector, Ap & Promega (Madison, WI) \\
pTR102 & Vector; Cm, Ap & Durland et al. 1990 \\
pRK2013 & Cloning vector, par; Tc & Weinstein et al. 1992 \\
pT2TX3K & mob+, tra+, helper plasmid, Km & Ditta 1986 \\
pT2TFX3K & pTR102 with $t f x B^{\prime} C D E F G, \mathrm{Km}$ & Triplett et al. 1994 \\
pFPMZ & pTR102 with $t f x A B C D E F G, \mathrm{Km}$ & Triplett et al. 1994 \\
\hline
\end{tabular}


tions were prepared by reading OD at $600 \mathrm{~nm}$ and plating 10fold serial dilutions on Bergensen's synthetic medium (Bergensen 1961) with the appropriate antibiotics. Antibiotic concentrations used were $50 \mu \mathrm{g} / \mathrm{ml}$ for ampicillin, kanamycin, and streptomycin, $100 \mu \mathrm{g} / \mathrm{ml}$ for cycloheximide, and $2.5 \mu \mathrm{g} / \mathrm{ml}$ for tetracycline.

\section{Construction of pFPMZ.}

To assess rhizosphere colonization and competition for nodulation of $R$. etli strain CE3, using different antibiotic resistances, we transferred pFPMZ into CE3 by conjugation as described by Triplett et al. (1989). This plasmid replicates in Rhizobium, is maintained without selection, contains a multiple cloning site, and expresses ampicillin resistance. This recombinant plasmid was developed by blunt ligating a $3.2-\mathrm{kb}$ KpnI-BamHI fragment from pTR102 containing the RK2 plasmid stability locus (Weinstein et al. 1992) into the unique SalI site of pFF1 (Durland et al. 1990), giving rise to pFPAR34. The multiple cloning site from pBluescript (Promega, Madison, WI), contained within a 645-bp HaeII fragment, was inserted into the unique EcoRI site of pFPAR34 by blunt end ligation. Sensitivity of CE3(pFPMZ) to TFX was shown with the assay by Triplett (1990).

\section{Growth chamber conditions.}

Plants of Phaseolus vulgaris cv. Pinto were grown in 1:1:1 sand/soil/vermiculite with sterile or nonsterile amended soil, in a growth chamber with $12 \mathrm{~h}$ of light at $24^{\circ} \mathrm{C}$ and $12 \mathrm{~h}$ of darkness at $22^{\circ} \mathrm{C}$. Characteristics of the Madison soil used in this study were previously reported by Bosworth et al. (1993). Inoculation treatments consisted of single strains and mixtures at different ratios of CE3(pFPMZ), a TFX-sensitive strain, and either CE3(pT2TX3K), TFX-nonproducing, TFX-resistant strain, or CE3(pT2TFXK), a TFX-producing strain, at a rate of $10^{7} \mathrm{CFU} / \mathrm{seed}$. The nominal ratio of the strains was estimated by plating aliquots of serial 10 -fold dilutions on the appropriate antibiotics.

\section{Determination of soil bacterial populations.}

Ten grams of soil from Madison was suspended in $90 \mathrm{ml}$ of water and an aliquot of this suspension was sonicated for $30 \mathrm{~s}$ in a water bath sonicator (model T-9B, manufactured by L \& $\mathrm{R}$, Kearney, NJ). Ten-fold serial dilutions were plated on $10 \%$ tryptic soybean agar amended with cycloheximide. Plates were incubated at $28^{\circ} \mathrm{C}$ for 4 days. The number of beannodulating rhizobia was estimated by the most probable number technique (Brockwell 1963).

\section{Determination of populations in the rhizosphere.}

Ninety-six hours after inoculation, the roots of five plants for each treatment were placed in $9 \mathrm{ml}$ of water, sonicated for $30 \mathrm{~s}$ in a water bath sonicator, diluted in a 10-fold manner, and plated on the appropriate antibiotics. Plates were incubated at $28^{\circ} \mathrm{C}$ for 3 days and counted (Araujo et al. 1994). Rhizosphere occupancy of each strain is shown as percentage of the total Rhizobium colonies. Rhizosphere populations in the nonsterile soil were not determined because we did not have a selective medium for Rhizobium. We used a one-sample Student's $t$ test to determine statistical differences between the representation of the strains in the inoculum and their proportion in the rhizosphere.

\section{Nodule occupancy assays.}

Three weeks after inoculation, 8 nodules from the crown area of the roots of 10 plants from each treatment were surfaced sterilized for $30 \mathrm{~s}$ in a solution of $30 \%$ household bleach, rinsed three times in sterile distilled water, placed individually in 96-well microtiter plates, crushed, and plated on differential antibiotics as described by Beattie and Handelsman (1989a). Plates were incubated for $48 \mathrm{~h}$ at $28^{\circ} \mathrm{C}$. Nodule occupancy is shown as proportion or percentage of nodules occupied by each strain. We used a one-sample Student's $t$ test to determine statistical differences between the representation of the strains in the inoculum and their nodule occupancy.

\section{Competitiveness analysis.}

To determine whether the presence of TFX production genes affected the level of rhizosphere colonization and competition for nodulation, we inoculated mixtures of TFXsensitive with TFX-nonproducing or TFX-producing strains at different ratios. This multiple-ratios approach allows determination of the ratio of the strains that results in a 1:1 representation in the rhizosphere or nodules. This sort of analysis has been previously described by Marques-Pinto et al. (1974), Amarger and Lobreau (1982), and Beattie and Handelsman (1989b).

\section{ACKNOWLEDGMENTS}

We thank Jennifer Jansen for technical assistance and James Borneman for critical reviews of this manuscript. This work has been supported by U.S. Department of Agriculture NRI Grant No. 94-37-050767 and Hatch Project No. 5201 from the University of Wisconsin-Madison College of Agriculture and Life Sciences.

\section{LITERATURE CITED}

Amarger N., and Lobreau, J. P. 1982. Quantitative study of nodulation competitiveness in Rhizobium strains. Appl. Environ. Microbiol. 44: 583-588.

Araujo, R. S., Robleto, E. A., and Handelsman, J. 1994. A hydrophobic mutant of Rhizobium etli altered in nodulation competitiveness and growth in the rhizosphere. Appl. Environ. Microbiol. 60:1430-1436.

Beattie, G. A., and Handelsman, J. 1989a. A rapid method for isolation and identification of Rhizobium from root nodules. J. Microbiol. Methods 9:29-33.

Beattie, G. A., and Handelsman, J. 1989b. Quantitative comparison of laboratory and field competitiveness of Rhizobium leguminosarum bv. phaseoli. Appl. Environ. Microbiol. 55:2755-2761.

Bergensen, F. J. 1961. The growth of Rhizobium in synthetic media. Aust. J. Biol. Sci. 14:349-360.

Beringer, J. E. 1974. R-factor transfer in Rhizobium leguminosarum. J. Gen. Microbiol. 84:188-198.

Bosworth, A. H., Breil, B. T., and Triplett, E. W. 1993. Production of the anti-rhizobial peptide, trifolitoxin, in sterile soils by Rhizobium leguminosarum bv. trifolii T24. Soil Biol. Biochem. 25:829-832.

Bottomley, P. J. 1992. Ecology of Bradyrhizobium and Rhizobium. Pages 293-348 in: Biological Nitrogen Fixation. G. Stacey, R. H. Burris, and H. J. Evans, eds. Chapman and Hall, New York.

Breil, B. T., Ludden, P. W., and Triplett, E. W. 1993. DNA sequence and mutational analysis of genes involved in the production and resistance of the antibiotic peptide trifolitoxin. J. Bacteriol. 175:3693-3702.

Breil, B. T., Triplett E. W., and Borneman, J. 1996. A newly discovered gene, $t f u A$, involved in the production of the ribosomally synthesized peptide antibiotic trifolitoxin. J. Bacteriol. 178:4150-4156.

Brockwell, J. 1963. Accuracy of plant-infection technique for counting populations of Rhizobium trifolii. Appl. Environ. Microbiol. 11:377383 . 
Ditta, G. 1986. Tn5 mapping of Rhizobium nitrogen fixation genes. Methods Enzymol. 118:519-528.

Dowling, D. N., and Broughton, W. J. 1986. Competition for nodulation of legumes. Annu. Rev. Microbiol. 40:131-157.

Durland, R. H., Toukdarian, F. F., and Helinski, D. R. 1990. Mutations in the $\operatorname{trf} A$ replication gene of the broad-host-range plasmid RK2 result in elevated plasmid copy numbers. J. Bacteriol. 172:3859-3867.

Lethbridge, B. J. 1989. The structure of trifolitoxin. Ph.D. thesis. University of Adelaide, Adelaide, Australia.

Maier, R. J., and Triplett, E. W. 1996. Toward more productive, efficient, and competitive nitrogen-fixing bacteria. Crit. Rev. Plant Sci. 15:191234.

Marques-Pinto, C., Phaik, Y., and Vincent, Y. M. 1974. Nodulation competitiveness amongst strains of Rhizobium meliloti and $R$. trifolii. Aust. J. Agric. Res. 25:317-329.

Noel, K. D., Vandenbosch, K. A., and Kulpaca, B. 1986. Mutations in Rhizobium phaseoli that lead to arrested development of infection threads. J. Bacteriol. 168:1392-1401.

Rossbach, S., Kulpa, D. A., Rossbach, U., and de Brujin, F. 1994. Molecular and genetic characterization of the rhizopine catabolism (mocABRC) genes of Rhizobium meliloti L5-30. Mol. Gen. Genet. 245:11-24

Schwinghamer, E. A., and Belkengren, R. P. 1968. Inhibition of rhizobia by a strain of Rhizobium trifolii: some properties of the antibiotic and of the strain. Arch. Mikrobiol. 64:130-145.

Streeter, J. G. 1994. Failure of inoculant rhizobia to overcome the domi- nance of indigenous strains for nodule formation. Can. J. Microbiol. 40:513-522.

Triplett, E. W. 1988. Isolation of genes involved in nodulation competitiveness from Rhizobium leguminosarum bv. trifolii T24. Proc. Natl. Acad. Sci. USA 85:3810-3814.

Triplett, E. W. 1990. Construction of a symbiotically effective strain of Rhizobium leguminosarum bv. trifolii with increased nodulation competitiveness. Appl. Environ. Microbiol. 56:98-103.

Triplett, E. W., and Barta, T. M. 1987. Trifolitoxin production and nodulation are necessary for the expression of superior nodulation competitiveness by Rhizobium leguminosarum bv. trifolii T24. Plant Physiol. 85:335-342.

Triplett, E. W., Breil, B. T., and Splitter, G. A. 1994. Expression of $t f x$ and sensitivity to the rhizobial peptide antibiotic trifolitoxin in a taxonomically distinct group of $\alpha$-proteobacteria including the animal pathogen Brucella abortus. Appl. Environ. Microbiol. 60:4163-4166.

Triplett, E. W., and Sadowsky M. J. 1992. Genetics for nodulation competition of legumes. Annu Rev. Microbiol. 46:399-428.

Triplett, E. W., Schink, J. M., and Noeldner, K. L. 1989. Mapping and subcloning of the trifolitoxin production and resistance genes from Rhizobium leguminosarum bv. trifolii T24. Mol. Plant-Microbe Interact. 2:202-208.

Weinstein, M., Roberts, R. C., and Helinski, D. R. 1992. A region of the broad-host range plasmid RK2 causes stable in planta inheritance of plasmids in Rhizobium meliloti cells isolated from alfalfa root nodules. J. Bacteriol. 174:7586-7589. 